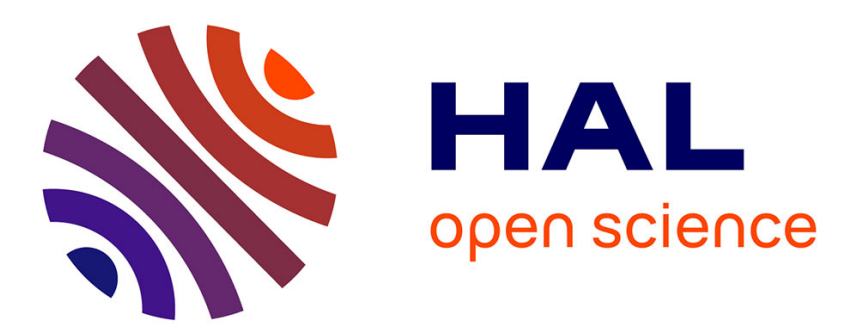

\title{
Surface fluctuations of liquids confined on flat and patterned solid substrates
}

Basile Pottier, Emilie Verneuil, Laurence Talini, Olivier Pierre-Louis

\section{To cite this version:}

Basile Pottier, Emilie Verneuil, Laurence Talini, Olivier Pierre-Louis. Surface fluctuations of liquids confined on flat and patterned solid substrates. Physical Review E : Statistical, Nonlinear, and Soft Matter Physics, 2014, 89 (5), pp.052403. 10.1103/PhysRevE.89.052403 . hal-01516017

\section{HAL Id: hal-01516017 https://hal.sorbonne-universite.fr/hal-01516017}

Submitted on 28 Apr 2017

HAL is a multi-disciplinary open access archive for the deposit and dissemination of scientific research documents, whether they are published or not. The documents may come from teaching and research institutions in France or abroad, or from public or private research centers.
L'archive ouverte pluridisciplinaire HAL, est destinée au dépôt et à la diffusion de documents scientifiques de niveau recherche, publiés ou non, émanant des établissements d'enseignement et de recherche français ou étrangers, des laboratoires publics ou privés. 


\title{
Surface fluctuations of liquids confined on flat and patterned solid substrates
}

\author{
Basile Pottier, ${ }^{1}$ Emilie Verneuil,${ }^{1}$ Laurence Talini, ${ }^{1},{ }^{*}$ and Olivier Pierre-Louis ${ }^{2, \dagger}$ \\ ${ }^{1}$ UPMC, CNRS, ESPCI Paris-Tech, UMR 7615, Laboratoire SIMM, 10 rue Vauquelin, F-75231 Paris Cedex 05, France \\ ${ }^{2}$ Institut Lumière Matière, UMR5306 Université Lyon 1-CNRS, Université de Lyon, F-69622 Villeurbanne Cedex, France
}

(Received 17 February 2014; published 19 May 2014)

\begin{abstract}
We report experimental measurements of the surface fluctuations of micron-thick oil films spread onto a solid substrate. We use a recently developed optical technique based on the measurement of the deflection of a laser beam triggered by changes in the local surface slope. When the liquid is spread on a flat substrate, fluctuation dynamics slow down as the thickness is decreased, in quantitative agreement with previous predictions. In addition, we investigate the consequences on surface fluctuations of the patterning of the substrate with a rectangular grating. For liquid film thicknesses smaller than the typical wavelength probed, we demonstrate that surface fluctuations are modified by the underlying pattern: The shape of the fluctuation spectra varies periodically with the spatial position over the pattern and, in addition, the fluctuations become locally anisotropic. However, the spatially averaged spectrum is isotropic.
\end{abstract}

DOI: 10.1103/PhysRevE.89.052403

PACS number(s): 68.03.Kn, 68.15.+e, 05.40.-a

\section{INTRODUCTION}

The measurement of the equilibrium fluctuations of a system provides information on its energetic and kinetic properties: The system reveals through its spontaneous thermal fluctuations. For soft matter systems, in which the energy scale is given by thermal energy $k T$ and which have a large susceptibility, the use of thermal noise to characterize mechanical properties is particularly relevant. In past decades, several experiments based on the measurements of fluctuations have been developed. For instance, a "zero applied stress rheometer" was built, in which the thermal fluctuations of a liquid result in fluctuations of the angular position of a cylindrical Couette apparatus [1]. Rheological properties can also be inferred from the Brownian motion of tracers added to a liquid, which is the principle of micro-rheology techniques [2]. Other experiments use the measurement of the fluctuations of free surfaces, which are corrugated owing to thermal energy [3-8]. The way a surface fluctuates not only reveals surface tension effects, but also the bulk rheological properties of the medium underneath. Characterizing the linear viscoelastic properties of fluids from their free surface fluctuations is particularly interesting for complex fluids in which an external forcing can induce heterogeneous flows $[9,10]$, making rheometrical measurements unsuited.

When a liquid film is deposited on a solid substrate, its free surface fluctuations can also provide information on the substrate. Actually, when the wavelengths of the probed fluctuation modes are smaller than the liquid depth, the surface fluctuations depend on both the thickness and the boundary condition at the solid substrate $[11,12]$. Therefore, when confinement is important, the measurement of the free surface fluctuations can be used to probe the underlying solid substrate.

Herein we study surface fluctuations of thin liquid films lying on solid substrates which are either flat or patterned. The effects of confinement on the free surface fluctuations of liquid films have been theoretically predicted [11,12]. Qualitatively,

\footnotetext{
*Corresponding author: laurence.talini@espci.fr

†olivier.pierre-louis@lpmcn.univ-lyon1.fr
}

such effects are expected to slow down the dynamics of fluctuations; for a given spatial mode, the average amplitude of the surface fluctuations, which is set by surface tension and/or elasticity, remains unchanged, but the amplitude of the spectrum is increased at low frequency and decreased at high frequency. Up to date, experimental studies considering confinement effects have dealt with complex liquids, namely polymer melts deposited onto a flat solid substrate $[13,14]$. For those systems as for simpler liquids, confinement induces a slowing down of the dynamics of the surface waves; however, when the polymer film thickness becomes comparable to the polymer radius of gyration, a behavior that has been identified as a suppression of fluctuation dynamics can arise. A recent study [15] has investigated the effect of confinement of polymer melts when the solid substrate is patterned. However, the fluctuations were spatially averaged over a distance about a hundred times larger than the pattern's period. Local effects on surface fluctuations by the substrate pattern have therefore never been investigated. In addition, specific effects arise from the viscoelastic properties of large molecular weight polymers, although they are generally not taken into account in the existing studies [14]; as a result, it is unclear to what extent the observed features of confinement depend on the complex nature of those systems. Experimental data on how confinement affects surface fluctuations are therefore needed for simpler systems, i.e., simple viscous liquids. In particular, in the case of a patterned substrate, a mapping of the fluctuation spectrum at a resolution much smaller than the pattern length scale would provide further information on the effect of the pattern.

Measurement of surface fluctuations is in itself a challenge since the amplitude of the resulting surface waves is of a few Angstroms only. Decades ago, experiments based on light scattering by liquid surfaces were developed [3]. They are limited to rather large surface fluctuations, and therefore are not well-suited for the measurement of confinement effects which decrease the amplitude of the fluctuation spectrum. Recent experiments on polymer films used x-ray photon correlation spectroscopy (XPCS) [5]; this technique is based on scattering by the liquid surface, and probes surface 
modes with wavelengths smaller than a few microns. Since confinement effects arise when the film thickness becomes smaller than the probed wavelength, those experiments are restricted to films with submicrometer thickness. Furthermore, at such small wavelengths, the associated time scales are short in ordinary liquids, and only very viscous liquids can be tested. XPCS is therefore not adapted for measurements with simple low-viscosity liquids.

Here we measure surface fluctuations of low-viscosity liquids with a technique based on the detection of local fluctuations in the surface slope of liquid surfaces [16]. Variations as small as $10^{-6}$ rad can be measured, which allows for the measurement of fluctuation modes with wavelength in the range 10-100 $\mu \mathrm{m}$. Confinement effects can thus be measured in much thicker liquid films as compared to previous studies. Note that the films are thick enough for the static properties of their free surface to be unaffected by the presence of the substrate. However, we demonstrate that the substrate influences the dynamics of surface fluctuations. The time scales we probe range from $10 \mu \mathrm{s}$ to $1 \mathrm{~s}$. We show that, for a flat substrate, confinement effects are well described by previous theoretical predictions. We also investigate the effect of a line-space grating with a rectangular cross section at the substrate surface. The typical wavelength of the probed surface modes is smaller than the period of the pattern, and the dynamics of surface waves are scanned in space along the pattern period. We observe spatially dependent dynamics, as well as anisotropic surface fluctuations of thin films lying on the grating. We show in particular that the spatially averaged spectra are isotropic.

\section{MATERIALS AND METHODS}

We have used flat unpolished silicon wafers, and patterned epoxy resin substrates with line-space gratings. The pattern was printed on a Plexiglas substrate using a micromiller, and molded with polydimethylsiloxane [(PDMS), Sylgard 184]. Once cured, the PDMS is grafted with a fluoro-silane and used as a mold for epoxy resin. The resin is further crosslinked by heating, and, at the end of the process, its surface presents a square-wave pattern with a period of $250 \mu \mathrm{m}$, and a height between tops and bottoms of $100 \mu \mathrm{m}$ (Fig. 1). We have checked that the chemical nature (either silicon or epoxy resin) of the solid substrate does not modify the surface fluctuations of the liquid, as expected within the range of liquid thickness we investigate; however, we show in the following that the fluctuations may strongly differ for flat and patterned substrates.

Two different oils of the same viscosity $\eta=0.1$ Pa.s have been used. The oil used with the flat substrate is a silicone oil (Rhodorsil v100) with surface tension $\gamma=20.5 \mathrm{mN} / \mathrm{m}$. The oil used with the patterned substrate is a specially designed oil (Cargille) whose refractive index matches that of the epoxy resin $(n=1.52)$. Its surface tension has been measured as $\gamma=38 \mathrm{mN} / \mathrm{m}$ from surface fluctuation measurements of a large depth sample [16].

Surface fluctuations are measured with a recently developed optical technique: surface fluctuation specular reflection (SFSR) spectroscopy in which a laser beam reflects off the liquid free surface; the reflected beam is deflected by surface

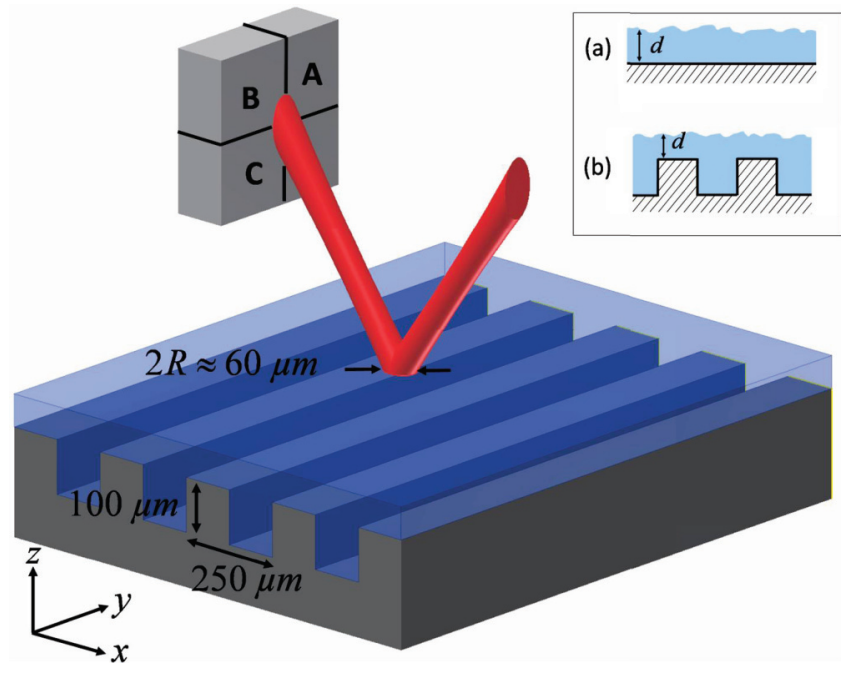

FIG. 1. (Color online) Schematic representation of the laser beam reflecting at the free surface of a liquid film lying on the pattern substrate. The reflected beam is centered on the four-quadrant photodiode, so that the fluctuations can be measured in the directions parallel (along $y$ ) and perpendicular (along $x$ ) to the grooves. $d$ is the depth of the liquid film for a flat substrate (a), or its depth above the tops of the patterned substrate (b).

fluctuations, and its fluctuating position can be related to the fluctuating local slope of the liquid surface [16]. Such a measurement mixes the contributions from several surface modes, unlike a light scattering experiment in which a single spatial mode is selected. Spatial resolution is therefore lost in the SFSR technique, but the resulting signal is much larger than in techniques based on scattering, thus allowing for measurements of surface fluctuations of much smaller amplitude. Consequently, SFSR has been successfully used to characterize materials with small surface fluctuations such as soft viscoelastic solids [7]. As detailed in the following, the contribution of each spatial mode to the SFSR signal is different, and the largest contribution results from the mode of wavelength close to the laser beam size. That size therefore sets the length scale of the measurement and, in the experiment described here, the beam radius is $R=28 \mu \mathrm{m}$.

Practically, the reflected beam is centered on a fourquadrant photodiode. The voltages delivered by three quadrants are used in order to measure the fluctuations along two different directions. Using the notations of Fig. 1, the difference between the voltages delivered by quadrants $\mathrm{A}$ and $\mathrm{B}$ provides a measurement of the fluctuations in the direction parallel to the grooves of the pattern, whereas we probe the surface fluctuations in the direction perpendicular to the grooves using quadrants B and C. The signals are amplified and digitized, and their power spectral densities (PSD) are further computed as the following:

$$
S(\omega)=\left\langle\frac{1}{T}\left|\mathrm{FT}\left(\delta V(t) / V_{\mathrm{tot}}\right)\right|^{2}\right\rangle_{\mathrm{ens}},
$$

where $\omega / 2 \pi$ is the frequency, $T$ is the duration of one acquisition and is set at $2.6 \mathrm{~s},\langle\rangle_{\mathrm{ens}}$ denotes the ensemble average, FT is the time Fourier transform, $\delta V(t)$ is either $V_{A}-V_{B}$ or $V_{B}-V_{C}$, and $V_{\text {tot }}$ is the total voltage delivered by 
the two quadrants whose difference is considered. Practically, the ensemble average is made over 100 acquisitions of duration $T$. Since, in each direction, we deal with signals that are normalized by the total intensity of the considered two quadrants, those signals can be compared even though the total intensity may slightly differ.

We have shown that, taking into account the contributions from all the spatial modes of the surface, the PSD $S(\omega)$ can be approximated by [16]

$$
S(\omega)=\frac{C}{\pi^{2} \lambda^{2}} \int_{0}^{\infty} P(q, \omega) q^{3} R^{2} e^{-\frac{q^{2} R^{2}}{c}} d q,
$$

where $C$ and $c$ are dimensionless numerical constants, $C=$ 0.966 and $c=1.658$, and $P(q, \omega)$ is the time Fourier transform of the autocorrelation function of the height of the surface. The other terms in the integral correspond to the weight of each surface mode of wave number $q$. As noted earlier, that weight is maximum for $q \approx 1 / R . P(q, \omega)$ depends on the properties of the probed interface whereas the other terms are set by the Gaussian laser beam parameters, i.e., the radius $R$ at the waist and laser wavelength $\lambda$.

Indeed, the signal $S(\omega)$ being normalized by the total voltage delivered by the quadrants, care must be taken in order that no parasitic light is received by the photodiode. In particular, reflection of the laser at the liquid-solid interface can interfere with light reflected at the liquid free surface. The mean roughness of the Si wafer (ca. $2 \mu \mathrm{m}$ ) is such that the light reflected at the liquid-solid substrate is scattered in all directions and we have checked that it has a negligible effect on the fluctuation signal. The very weak reflection at the liquidsolid interface nevertheless allowed for the measurement of the film thickness using an optical profilometer. As shown in the next section, the use of a rough substrate therefore allowed demonstration of the full agreement between experimental data and theoretical predictions made for liquids lying on flat substrates, provided the film thickness remained larger than the substrate roughness. In the experiments with the patterned substrate, the liquid and substrate are index matched; there is therefore no reflection at the liquid-substrate interface. Therefore, in the latter experiments, the film thickness could not be directly determined but we show in the following that it could be inferred from the fluctuation spectra obtained above the top of the patterns. All experiments were performed at $T=25^{\circ} \mathrm{C}$.

\section{FLAT SUBSTRATE}

We first present results obtained with the liquid film of thickness $d$, spread onto a flat solid surface. The dynamics of the surface fluctuations have been theoretically predicted in that case $[11,12]$. Before describing the experimental data, we briefly recall those predictions and we further derive the expected behavior of $S(\omega)$ at low and high frequencies. For a simple fluid of density $\rho$, viscosity $\eta$, and surface tension $\gamma$, the average magnitude of the thermally activated waves is set by surface tension; the mean surface roughness is given by $\left\langle h^{2}\right\rangle=k_{B} T \ln \left(q_{\max } l_{c}\right) /(2 \pi \gamma)$ where $1 / q_{\max }$ is an atomic cutoff length, $l_{c}$ is the capillary length, and $l_{c}=\sqrt{\gamma / \rho g}$ with $g$ the acceleration of gravity. For the liquids considered herein, the thermally induced surface roughness is of a few
Angstroms, and indeed the associated flow has a very small Reynolds number. It is given by $\operatorname{Re} \approx \rho L\left\langle h^{2}\right\rangle^{1 / 2} \omega / \eta$ where $L$ is the vertical length over which the velocity vanishes. Depending on the film confinement, $L$ is either given by the inverse wave number $1 / q$ for large film thickness $(q d \gg 1)$ or by $d$ for thin films $(q d \ll 1)$. At the length scale probed $1 / q \approx R$ ( $R$ being the beam radius), the Reynolds number is at most $10^{-7}$, and the convective term of the Navier-Stokes equation is therefore negligible. The nonstationary term in the Navier Stokes equation can be neglected provided the Stokes number St $\approx \rho \omega L^{2} / \eta$ is small, i.e., for frequencies $\omega / 2 \pi \ll 20 \mathrm{kHz}$ within our experimental conditions. The Stokes equation can then be used to describe the flow within the liquid, and the capillary surface waves are overdamped. The resolution of the Stokes equation with the proper boundary conditions, which is fully detailed in Refs. [11,12] yields the surface mechanical susceptibility. For a system in thermal equilibrium, the susceptibility is itself related to $P(q, \omega)$ through a fluctuation dissipation relation, which leads to [12]:

$$
P(q, \omega)=\frac{k_{B} T}{\pi \gamma q^{2}} \frac{\frac{1}{\tilde{\omega}}}{1+\left(\frac{\omega}{\tilde{\omega}}\right)^{2}} .
$$

In the overdamped regime considered herein, the dispersion equation has purely imaginary roots; $\tilde{\omega}$ corresponds to the opposite of the imaginary part of that root and is such that $\tilde{\omega}=\gamma q^{2} / \eta B(q)$, where function $B(q)$ depends on the film thickness $d$ [12]:

$$
B(q)=4 q \frac{\left(\cosh ^{2}(q d)+(q d)^{2}\right)}{(\sinh (2 q d)-2 q d)} .
$$

The signal measured by SFSR technique results from the contributions of several surface modes and its full expression is therefore given by the integral expression of Eq. (2), together with Eq. (3). In the following, we derive simple scaling laws for $S(\omega)$ by using simplified dispersion equations in the limit of large and small thicknesses.

Without confinement, that is, for $q d \gg 1$, the dispersion equation becomes $\tilde{\omega}=\gamma q / 2 \eta$, yielding a low-frequency regime where $S(\omega)$ is independent of frequency: for $\omega \ll \tilde{\omega}$ : $S(\omega) \propto k_{B} T \eta R / \lambda^{2} \gamma^{2}$. In the high-frequency regime, i.e., $\omega \gg \tilde{\omega}$ the signal decreases, varying as the inverse of the squared frequency: $S(\omega) \propto k_{B} T / \lambda^{2} \eta R \omega^{2}$. A Lorentzian shape is therefore expected for $S(\omega)$ without confinement effects, i.e., for a medium of depth $d \gg R$.

When the liquid film is confined, i.e., in the limit $q d \ll 1$, the dispersion equation becomes $\tilde{\omega}=\gamma q^{4} d^{3} / 3 \eta$. At small frequencies, no simple variation law can be derived for $S(\omega)$, since the contributions of different spatial modes are mixed. Actually, in that frequency range the effect of confinement strongly differs according to the spatial mode since $\tilde{\omega}$ varies with $q^{4}$ : The modes with smaller wave vectors are more affected by confinement than the modes of larger wave vectors. The signal is thus expected to decrease with increasing frequency, but more slowly than in the high-frequency regime in which $S(\omega) \propto 1 / \omega^{2}$. In the high-frequency regime, the signal reduces to $S(\omega) \propto k_{B} T d^{3} / \lambda^{2} \eta R^{4} \omega^{2}$.

In summary, one expects to measure significant effects of confinement with SFSR technique provided the film thickness is smaller than the beam size. In that case, the signal becomes 

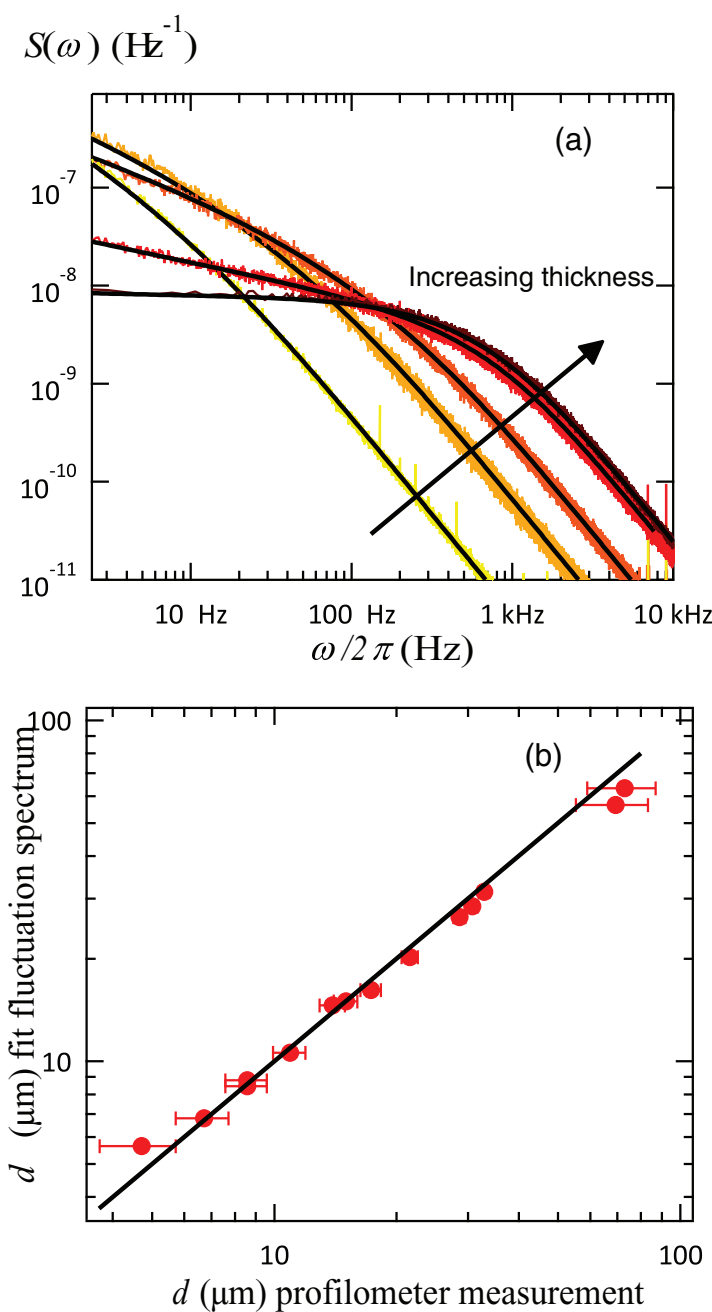

FIG. 2. (Color online) (a) Fluctuation spectra of thin silicone oil films on a flat solid substrate. The experimental data (colors) corresponds to different film thicknesses and their respective fits to the theoretical expression (solid black lines), with the thickness of the film, $d$ as the only fitting parameter. The inferred thicknesses are from bottom to top at high frequencies: $5.3 \mu \mathrm{m}, 8.4 \mu \mathrm{m}, 14.6 \mu \mathrm{m}$, and $63.2 \mu \mathrm{m}$. The spectrum in darker color is obtained with a liquid of depth $1 \mathrm{~mm}$ and is fit to the expression for infinite thickness media. (b) Film thickness obtained by fitting the experimental spectra vs film thickness directly measured using a profilometer. The error bars result from the difficult setting of the profilometer, the reflection at the substrate being very weak. The vertical error bars are too small to appear. The solid line represents $d \_$fit $=d \_$profilometer.

dependent on the film thickness; in particular, at high frequency the signal is expected to strongly decrease with decreasing thickness since $S(\omega) \propto d^{3}$.

Figure 2(a) shows the different spectra measured with silicone oil films of thicknesses ranging from $5.3 \mu \mathrm{m}$ to $1 \mathrm{~mm}$. As expected, when the film thickness is much larger than the beam size at the surface, $d=1 \mathrm{~mm} \gg R=28 \mu \mathrm{m}$, the spectrum is well described by a Lorentzian. For a thickness $d=63.2 \mu \mathrm{m}$, the spectrum slightly deviates from a Lorentzian shape. For smaller film thicknesses, the spectrum is shifted toward lower frequencies as the dynamics is slowed down. Since the liquid properties are known, a fit to each spectrum can be performed with the film thickness as the only fitting parameter. In each case, the fit to the experimental data is excellent. We have reported the obtained adjusted thicknesses as a function of the ones independently measured with the profilometer in Fig. 2(b). Note that, owing to the micrometric roughness of the substrate, we do not report data for films thinner than $5 \mu \mathrm{m}$, since their thickness is ill defined. Within the investigated thickness range, the values are in excellent agreement for film thicknesses spanning more than one decade, which validates for the first time the predictions made on the effect of confinement on thermal fluctuation of thin films in that range. As a final remark, we have checked that, whatever the film thickness, the fluctuation spectrum is isotropic, i.e., it does not depend on the direction that is probed. Isotropic surface fluctuations are indeed expected for a film lying on a flat substrate, but we show in the following section that anisotropic fluctuations can be observed with a patterned substrate.

\section{PATTERNED SUBSTRATE}

We now turn to the results obtained with oil films spread onto line-space gratings of the rectangular cross section. For films thinner than ca. $30 \mu \mathrm{m}$, the spectra exhibit a dependence on both the position $x$ of the beam relative to the pattern and on the direction along $(y)$ or across $(x)$ the pattern.

We first focus on the surface fluctuations along the direction $y$ parallel to the lines of the pattern. Figure 3(a) shows the spectra obtained at different $x$ positions above the pattern. The effect of confinement varies periodically along the pattern. The amplitude at large frequencies is maximum above the thickest region of the liquid film and decreases away from that position. In Fig. 3(b) we have reported the signal value taken at a frequency of $2 \mathrm{kHz}$ as a function of the position $x$ across the pattern, for liquid films of different thicknesses. The signal is scaled by its maximal value along the pattern. The variations obtained in Fig. 3(b) depend on the film thickness: Whereas the signal is flat for a film thickness $d=40 \mu \mathrm{m}$, it follows the pattern for thinner films.

We find that the spectra above the tops of the pattern are well described using the expressions derived for a flat substrate [see Fig. 3(c)]; the film thickness above the pattern tops can thus be estimated by performing a fit to the experimental spectra following Eq. (2) with Eq. (3). The obtained thickness values are reported in the caption of Fig. 3(b).

In contrast, as illustrated in Fig. 3(c), the profile of the spectra obtained above the grooves cannot be properly described using the predictions for a film of same local thickness lying on a flat substrate. This shows that above the trenches, the confinement in the $x$ direction modifies the fluctuation spectrum as well. A quantitative description of this effect is yet to be derived. Indeed, the surface fluctuations are then sensitive to the zero velocity boundary condition on the vertical walls of the grooves, and a computation taking into account those conditions is needed to quantitatively predict the fluctuations.

As noted earlier, the spectra also exhibit a dependence on the measurement direction. Figure 4(a) shows the spectra obtained above the tops and above the bottoms of the pattern, in both the parallel and perpendicular directions. First, let us focus on the measurements above the tops of the pattern 


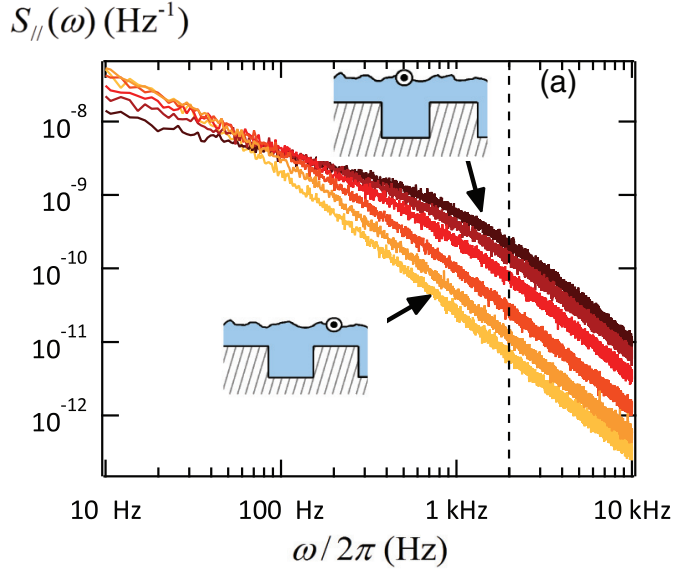

(b)

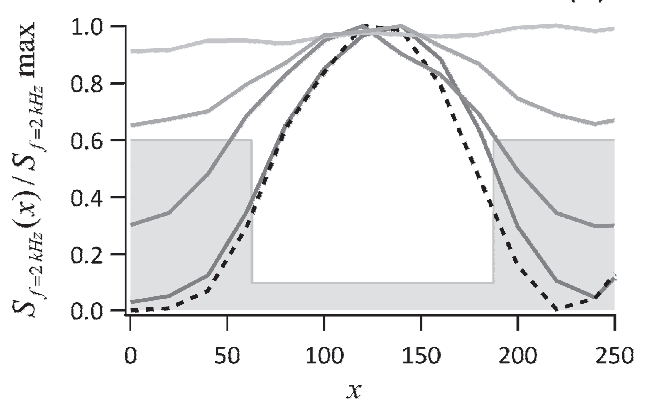

(displacement perpendicular to the grooves in $\mu \mathrm{m}$ )

$S_{/ /}(\omega)\left(\mathrm{Hz}^{-1}\right)$

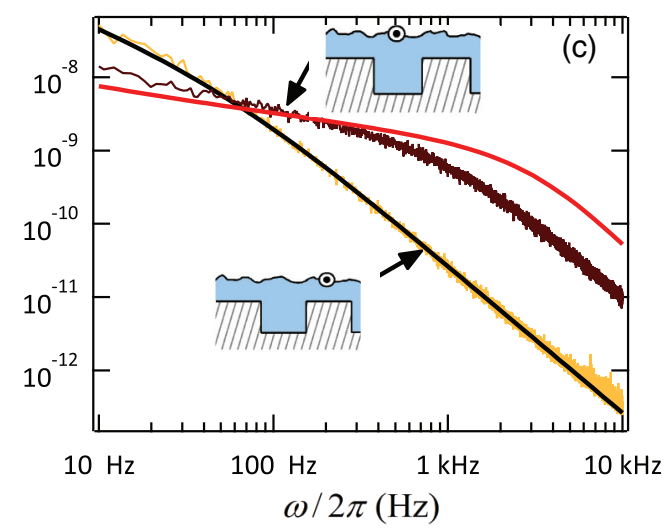

FIG. 3. (Color online) (a) Fluctuation spectra of an oil film on a line-space grating. Measurements are taken in the parallel direction and at different locations along the $x$ direction scanning a period of the pattern $(250 \mu \mathrm{m})$. The step in the position is $\Delta x=20 \mu \mathrm{m}$. The dotted line indicates the frequency corresponding to the spectrum values reported in Fig. 3(b). (b) Spectrum value at $2 \mathrm{kHz}$ normalized by its maximum value as a function of the displacement $x$ perpendicular to the grooves. The data correspond to different thicknesses above the top of the square-wave patterned substrate (thickness values resulting from the fit of the spectra above the tops): dotted line $2.3 \mu \mathrm{m}$, solid lines (from the darker to the lighter) $4.5 \mu \mathrm{m}$ (darker gray), $13 \mu \mathrm{m}$, $30 \mu \mathrm{m}$, and $40 \mu \mathrm{m}$. The gray area represents the pattern of the substrate. (c) Same data as Fig. 3(a) but only the spectra above, respectively, a top and a groove are shown. The theoretical spectra expected for a film lying on a flat substrate and of same local thickness (respectively, $4.5 \mu \mathrm{m}$ and $104.5 \mu \mathrm{m}$ ) are shown in solid lines. The agreement is very good for the spectrum above a top but there is a discrepancy for the spectrum obtained above a groove.
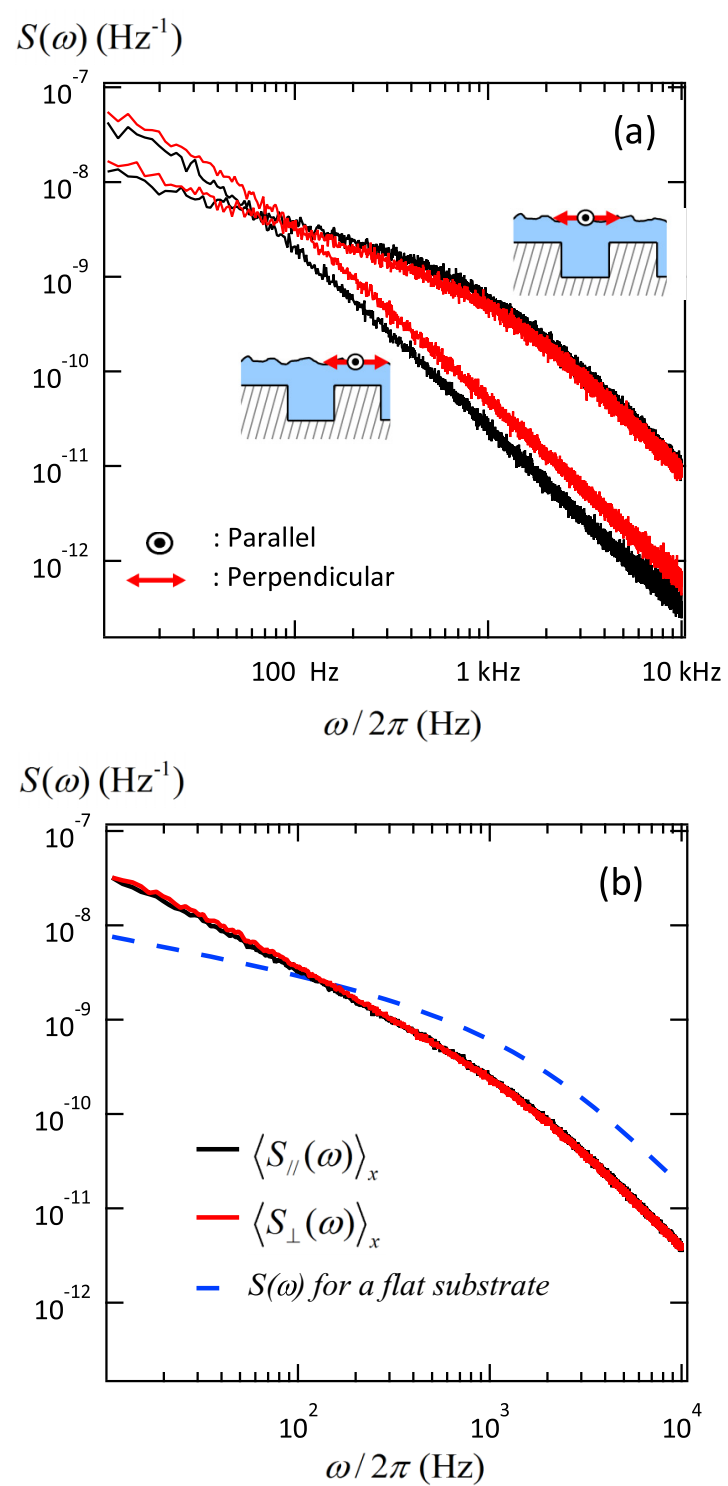

FIG. 4. (Color online) (a) Surface fluctuation spectra of a film of thickness $4.5 \mu \mathrm{m}$ in the directions parallel (black lines) and perpendicular [red (gray) lines] to the lines of the patterned substrate. As schematized, two positions are shown: above the center of the pattern tops (lower spectra at high frequency), and above the pattern bottoms. At both locations, the spectra differ, showing the anisotropy of the fluctuations resulting from the patterned substrate. (b) Spectra averaged over a spatial pattern period in the parallel (black) and perpendicular [red (gray)] directions. The spectra superpose but they cannot be described using the results on flat substrates. In particular, they significantly differ from the expected spectrum of a liquid film lying on a flat substrate and of the same average thickness, i.e., $d=$ $52.25 \mu \mathrm{m}$ (dashed line).

where the spectra were found to fit to the prediction made on flat surfaces. The spectrum in the parallel direction is found to be shifted to lower frequencies as compared to the one in the direction across. Such a shift indicates that, above the tops of the pattern, confinement is stronger in the parallel direction compared to the cross direction. The smaller confinement effect in the perpendicular direction can 
be understood intuitively as the consequence of the presence of the edges of the pattern top, beyond which the liquid is less confined. The opposite effect is observed above the pattern valleys since confinement is then qualitatively slightly larger in the perpendicular direction. Except for the spectrum obtained above the tops in the parallel direction, the consequences of confinement cannot be accounted for using the expression for a flat substrate with an effective depth as a free-fitting parameter. The more complex boundary conditions at the patterned surface, which include zero-velocity conditions on the vertical walls, should be taken into account in order to properly describe those effects.

The spectrum averaged over one spatial period of the pattern [Fig. 4(b)] exhibits two important properties: (i) It is isotropic, leading to identical spectra for perpendicular and parallel measurements, and (ii) it cannot be fitted with the theoretical prediction for a flat substrate with an effective average thickness. These unexpected and nontrivial results call for further modeling.

Our results with patterned substrates are consistent with a previous study [15], in which ultrathin polymer films were deposited on line-space gratings and their free surface fluctuations were measured using XPCS. The beam size was much larger than the pattern's period, leading to measurements averaged about a hundred periods. For wavelengths ranging from the pattern wavelength to a cutoff wavelength, no anisotropy was observed in agreement with our data. However, at wavelengths larger than the cutoff wavelength, the study interestingly revealed anisotropic fluctuations. The anisotropy was attributed to the combination of the film curvature, resulting from the van der Waals interactions with the substrate, and the strong confinement of polymers over the pattern tops, where the film thickness was smaller than the gyration radius. Both effects being absent in our system, it is clear that we should not expect a long wavelength anisotropy from a similar origin. Therefore, our results are essentially consistent with those of [15]. In addition, using a beam size roughly 10 times smaller than the pattern periodicity, we observe for the first time that fluctuations are space dependent and anisotropic at wavelengths smaller than the pattern periodicity.

\section{CONCLUSION}

We have studied the effect of confinement on the fluctuations of the free surface of viscous liquids. We have used a recently developed optical technique, well suited for the dynamical measurement of fluctuations at a liquid surface in a large frequency range. The slowing down of surface waves for liquid films on flat substrates is quantitatively described using previous theoretical predictions with a remarkable agreement. When the liquid film lies upon a line-space grating, we evidence original effects. First, the fluctuation spectra become position dependent along the pattern's period and, second, the surface fluctuates anisotropically. However, we find that the spatially averaged spectrum is isotropic.

\section{ACKNOWLEDGMENT}

We thank A. Prevost for his help in the imprinting of the Plexiglas substrate.
[1] L. Bellon, L. Buisson, S. Ciliberto et al., Rev. Sci. Instrum. 73, 3286 (2002).

[2] P. Cicuta and A. M. Donald, Soft Matter 3, 1449 (2007).

[3] D. Langevin, Light Scattering by Liquid Surfaces and Complementary Techniques (Marcel Dekker, New York, 1992).

[4] D. M. A. Buzza, Langmuir 18, 8418 (2002).

[5] R. L. Leheny, Current Opinion in Colloid \& Interface Science 17, 3 (2012).

[6] T. Mitsui and K. Aoki, Phys. Rev. E 80, 020602(R) (2009).

[7] B. Pottier, G. Ducouret, C. Fretigny et al., Soft Matter 7, 7843 (2011).

[8] C. Pigot and A. Hibara, Anal. Chem. 84, 2557 (2012).

[9] S. Q. Wang, Adv. Polymer Sci. 138, 227 (1999).
[10] T. Divoux, D. Tamarii, C. Barentin, and S. Manneville, Phys. Rev. Lett. 104, 208301 (2010).

[11] J. Jackle, J. Phys. Condens. Matter 10, 7121 (1998).

[12] M. L. Henle and A. J. Levine, Phys. Rev. E 75, 021604 (2007).

[13] H. Kim, A. Rühm, L. B. Lurio, J. K. Basu, J. Lal, D. Lumma, S. G. J. Mochrie, and S. K. Sinha, Phys. Rev. Lett. 90, 068302 (2003).

[14] Z. Jiang, H. Kim, X. Jiao et al., Phys. Rev. Lett. 98, 227801 (2007).

[15] K. J. Alvine, Y. Dai, H. W. Ro, S. Narayanan, A. R. Sandy, C. L. Soles, and O. G. Shpyrko, Phys. Rev. Lett. 109, 207801 (2012).

[16] A. Tay, C. Thibierge, D. Fournier et al., Rev. Sci. Instrum. 79, 103107 (2008) 